\title{
Leptonic and Charged Kaon Decay Modes of the $\phi$ Meson Measured in Heavy-Ion Collisions at the CERN Super Proton Synchrotron
}

D. Adamová, ${ }^{1}$ G. Agakichiev, ${ }^{2}$ D. Antończyk, ${ }^{2}$ H. Appelshäuser,${ }^{3}$ V. Belaga, ${ }^{4}$ J. Bielčíková,,${ }^{5} 11$ P. Braun-Munzinger, ${ }^{2}$ O. Busch, ${ }^{2}$ A. Cherlin, ${ }^{6}$ S. Damjanovic, ${ }^{5}$ T. Dietel,${ }^{5}$ L. Dietrich,${ }^{5}$ A. Drees, ${ }^{7}$ S. I. Esumi, ${ }^{5}$ K. Filimonov, ${ }^{5}$ K. Fomenko, ${ }^{4}$ Z. Fraenkel, ${ }^{6}$ C. Garabatos, ${ }^{2}$ P. Glässel, ${ }^{5}$ G. Hering, ${ }^{2}$ J. Holeczek, ${ }^{2}$ G. Krobath,${ }^{5}$ V. Kushpil,${ }^{1}$ W. Ludolphs, ${ }^{5}$ A. Maas, ${ }^{2}$ A. Marín, ${ }^{2}$ J. Milošević, ${ }^{5}$ D. Miśkowiec, ${ }^{2}$ R. Ortega, ${ }^{5}$ Y. Panebrattsev, ${ }^{4}$ O. Petchenova, ${ }^{4}$ V. Petráček, ${ }^{5}$ S. Radomski, ${ }^{2}$ J. Rak, ${ }^{2}$ I. Ravinovich, ${ }^{6}$ P. Rehak, ${ }^{8}$ H. Sako, ${ }^{2}$ W. Schmitz, ${ }^{5}$ J. Schukraft, ${ }^{9}$ S. Sedykh, ${ }^{2}$ S. Shimansky, ${ }^{4}$ J. Stachel, ${ }^{5}$ M. Šumbera, ${ }^{1}$ H. Tilsner, ${ }^{5}$ I. Tserruya,${ }^{6}$ G. Tsiledakis, ${ }^{2}$ J. P. Wessels,${ }^{10}$ T. Wienold,${ }^{5}$ J. P. Wurm, ${ }^{11}$ S. Yurevich, ${ }^{5}$ and V. Yurevich ${ }^{4}$

(CERES Collaboration)

${ }^{1}$ NPI/ASCR, Řě, Czech Republic

${ }^{2}$ GSI, Darmstadt, Germany

${ }^{3}$ Universität Frankfurt, Frankfurt, Germany

${ }^{4} J I N R$, Dubna, Russia

${ }^{5}$ Universität Heidelberg, Heidelberg, Germany

${ }^{6}$ Weizmann Institute, Rehovot, Israel

${ }^{7}$ Department for Physics and Astronomy, SUNY, Stony Brook, New York, USA

${ }^{8}$ Brookhaven National Laboratory, Upton, New York, USA

${ }^{9}$ CERN, Geneva, Switzerland

${ }^{10}$ Universität Münster, Germany

${ }^{11}$ Max-Planck-Institut für Kernphysik, Heidelberg, Germany

(Received 3 December 2005; published 21 April 2006)

\begin{abstract}
We report on results of a measurement of $\phi$ meson production in central $\mathrm{Pb}-\mathrm{Au}$ collisions at $E_{\mathrm{lab}}=$ $158 \mathrm{~A} \mathrm{GeV}$. For the first time in the history of high energy heavy-ion collisions, $\phi$ mesons were reconstructed both in the $K^{+} K^{-}$and the dilepton decay channels in the same experiment. This measurement yields rapidity densities near midrapidity, from the two decay channels, of $2.05 \pm$ 0.14 (stat) \pm 0.25 (syst) and $2.04 \pm 0.49$ (stat) \pm 0.32 (syst), respectively. The shape of the measured transverse momentum spectrum is also in close agreement in both decay channels. The data rule out a possible enhancement of the $\phi$ yield in the leptonic over the hadronic decay channel of a factor 1.6 or larger at the $95 \%$ C.L. This rules out the discrepancy reported in the literature between measurements of the hadronic and dimuon decay channels by two different experiments.
\end{abstract}

DOI: 10.1103/PhysRevLett.96.152301

PACS numbers: 25.75.Dw, 13.85.Ni, 13.85.Qk

In ultrarelativistic nucleus-nucleus collisions matter is created at high temperature and density. For top super proton synchrotron (SPS) energies and above, the corresponding fireball is very likely in a deconfined state [1] as expected from solutions of quantum chromodynamics (QCD) on a space-time lattice [2]. Detailed studies of hadron abundances imply that their yield is frozen very close to the predicted phase boundary between confined and deconfined matter [3]. Strangeness enhancement has been suggested as a signature of a deconfined stage [4]. In this context it is important to understand the production of $\phi$ mesons which carry hidden strangeness. Furthermore, near the phase boundary the $\phi$ meson mass, width, and its branching ratios into kaons and leptons might be modified $[5,6]$. Because of final state interactions of the kaons, such a modification would probably only be visible in the lepton decay channel, which could result in different $\phi$ yields depending on which decay channel is studied [7]. Indeed, the experimental situation concerning $\phi$ production at the top SPS energy provides hints for such a scenario as the NA50 Collaboration reported a $\phi$ yield measured via dimuons [8] which exceeded the yield determined by NA49 in the $K^{+} K^{-}$channel [9] by factors between two and four [10] in the common transverse momentum range. Further, the $m_{t}$ spectra exhibit a different inverse slope parameter [10], $305 \pm 15 \mathrm{MeV}$ in NA49 and $218 \pm$ $6 \mathrm{MeV}$ in NA50, fitted in their $m_{t}$ acceptance regions.

The upgrade of the CERES experiment [11-14] makes it possible for the first time to simultaneously study the leptonic and the charged kaon decay modes of the $\phi$ meson at the SPS, thus shedding light onto the $\phi$ puzzle. In this Letter, we present results of $\phi$ mesons reconstructed both in the charged kaon $\left(K^{+} K^{-}\right)$and in the dilepton $\left(e^{+} e^{-}\right)$ decay mode. The kaon (dilepton) analysis uses 24 (18) $1.5 \times 10^{6} 158 \mathrm{~A} \mathrm{GeV} / \mathrm{c} \mathrm{Pb}$ on $\mathrm{Au}$ collisions taken at the most central $7 \%$ of the geometrical cross section. 
The CERES experiment is optimized to measure low mass electron pairs close to midrapidity $(2.1<\eta<2.65)$ with full azimuthal coverage [15-19]. A vertex telescope, composed of two silicon drift detectors (SDD) positioned at $10 \mathrm{~cm}$ and $13.8 \mathrm{~cm}$ downstream of a segmented Au target (thickness equivalent to $1.33 \%$ interaction length), provides a precise vertex reconstruction. Two Ring Imaging Cherenkov (RICH) detectors, operated at a high threshold $\left(\gamma_{\text {th }}=32\right)$, provide electron identification in a large hadronic background. The new radial-drift time projection chamber (TPC), positioned downstream of the original spectrometer inside a magnetic field, provides the momentum of charged particles with a resolution $\Delta p / p \sim$ $\left\{(2 \%)^{2}+[1 \% \cdot p(\mathrm{GeV} / c)]^{2}\right\}^{1 / 2}$ and additional electron identification via $d E / d x$. Charged particles from the target are reconstructed by matching track segments in the SDD and in the TPC using a momentum-dependent matching window. Matching to a ring in the RICH is also required for the dilepton analysis.

To study the $\phi$ meson in the $K^{+} K^{-}$decay mode, all charged particles get assigned the kaon mass (no particle identification is used). Only a conservative upper cut in the $d E / d x$ signal (corresponding to $90 \%$ of the Fermi plateau value) for momenta between $1.25 \mathrm{GeV} / c$ and $4 \mathrm{GeV} / c$, suppressing $83 \%$ of the electrons, is applied to enhance the kaon content of the sample. Tracks in the TPC are required to contain more than 12 hits out of a maximum 20 possible, to ensure good momentum resolution. Tracks in the geometrical acceptance $0.13 \mathrm{rad}<\theta<0.24$ rad with a transverse momentum $p_{t}$ larger than $0.25 \mathrm{GeV} / c$ are selected. To reduce the contamination from other particle species, cuts in the Podolanski-Armenteros parameter [20] and in the opening angle between the kaons are applied [21]. The $\phi$ meson in the kaon decay mode is studied in the rapidity interval $2.0<y^{\phi}<2.4$ for $p_{t}^{\phi}>0.75 \mathrm{GeV} / c$.

To obtain the $\phi$ transverse momentum spectrum the invariant-mass distributions of $K^{+} K^{-}$pairs were accumulated in $\left(p_{t}^{\phi}, y^{\phi}\right)$ bins. The invariant-mass distributions of the combinatorial background are calculated using the mixed-event technique for each $\left(p_{t}^{\phi}, y^{\phi}\right)$ bin. One such invariant-mass distribution, after background subtraction, is presented in Fig. 1. Residual background appears in the low $p_{t}^{\phi}$ bins. The yield of the $\phi$ mesons is, therefore, determined by fitting a relativistic Breit-Wigner distribution with parameters taken from the Particle Data Group compilation [22] (convoluted with the experimental resolution function obtained by a Monte Carlo simulation) superimposed on a linear background, to the measured mass spectrum. The signal to background ratio varies from $1 / 2000$ to $1 / 180$ with increasing $p_{t}^{\phi}$. The signal is integrated in the mass range between $1.0 \mathrm{GeV} / c^{2}$ and $1.05 \mathrm{GeV} / c^{2}$. The resulting $\phi$ yields need to be corrected for acceptance and efficiency.

To study the $\phi$ meson in the dilepton $\left(e^{+} e^{-}\right)$decay mode, electrons are identified among all charged hadrons using the RICH detectors and the $d E / d x$ signal in the TPC.

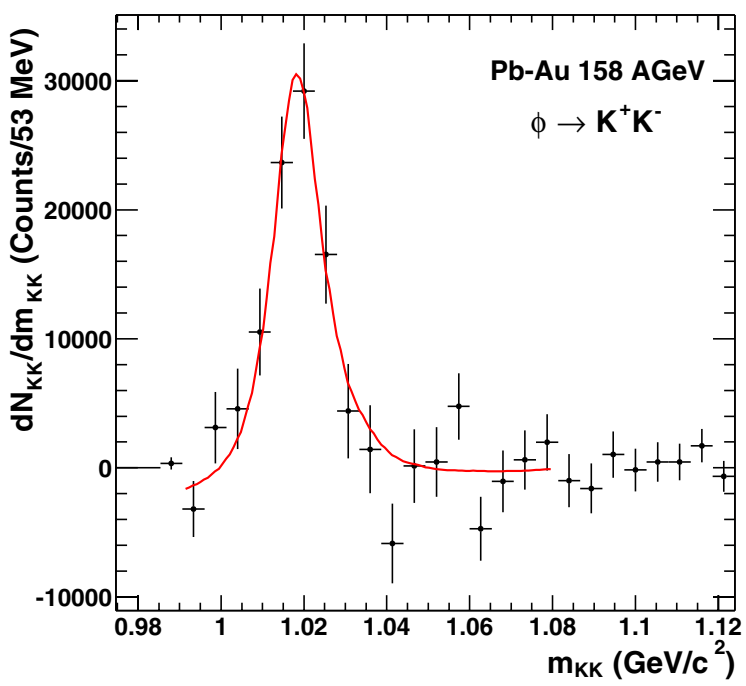

FIG. 1 (color online). Invariant-mass spectra of $K^{+} K^{-}$pairs after background subtraction for the $\left(p_{t}^{\phi}, y^{\phi}\right)$ bin $1.5 \mathrm{GeV} / c<$ $p_{t}^{\phi}<1.75 \mathrm{GeV} / c$ and $2.2<y^{\phi}<2.4$.

The two RICH detectors are used in a combined mode. Cherenkov rings with asymptotic radii are identified using a Hough transformation [see section 3.2.4 in Ref. [18] ]. A pion rejection factor of 2000 is achieved in the RICH [23] for an electron efficiency of 0.70 with the quality cuts applied in the analysis [24,25]. The TPC electron selection is done based on the $d E / d x$ signal and its resolution. The combined pion rejection factor varies from $4 \times 10^{4}$ to $1.8 \times 10^{4}$ for momenta between $1 \mathrm{GeV} / c$ and $2.5 \mathrm{GeV} / c$ for total electron efficiencies of $68 \%$ and $66 \%$ [24], respectively.

The main difficulties of reconstructing $\phi$ mesons in the dielectron channel are the low branching ratio of this decay mode and the large amount of combinatorial background from $\gamma$ conversions and from Dalitz decays of neutral mesons. Therefore, besides a very good electron identification, removal of electron pairs from $\gamma$ conversions and $\pi^{0}$ Dalitz decays from the sample is vital to reduce the combinatorial background. The details of the rejection strategy are explained in [14,24-26]. Electron tracks in the geometrical acceptance $0.14 \mathrm{rad}<\theta<$ $0.243 \mathrm{rad}$ and with a transverse momentum $p_{t}>$ $0.2 \mathrm{GeV} / c$ are selected [24,25]. The $\phi$ meson in the dilepton decay mode is studied in the rapidity interval $2.1<y^{\phi}<2.65$. The signal in the $e^{+} e^{-}$channel is obtained from the invariant-mass distributions of unlike-sign pairs after full rejection and subtraction of the mixed-event combinatorial background. The mixed-event background is normalized to the like-sign pair background in the mass region $0.2 \mathrm{GeV} / c^{2}<m_{e^{+} e^{-}}<1.6 \mathrm{GeV} / c^{2}$. The integrated yield in the mass range between 0.9 and $1.1 \mathrm{GeV} / c^{2}$ is $229 \pm 53$ with a signal to background ratio of $1 / 12$ and needs to be corrected for acceptance, reconstruction efficiency and physics background under the $\phi$ peak. 
In order to determine the acceptance, decay and efficiency corrections, GEANT [27] simulations containing the description of the CERES experiment are used. The Monte Carlo simulation is tuned to reproduce all aspects of the data $[14,24,25]$. Sources of $\phi$ mesons with realistic transverse momentum and rapidity distributions were embedded into real events to simulate the background. The simulation of the dilepton channel is done using the GENESIS simulation code [28]. All $\left(p_{t}^{\phi}, y^{\phi}\right)$ bins with an acceptance larger than 0.5 are used in the $K^{+} K^{-}$analysis. The reconstruction efficiency is of the order of 0.4 with a slight dependence on the transverse momentum, given by the decay in flight of the charged kaons, the pair cuts applied, and the single track efficiency. The acceptance for the $e^{+} e^{-}$pairs varies from 0.165 to 0.185 in the measured rapidity range and the reconstruction efficiency after full rejection varies from 0.22 to 0.145 with the $\phi$ transverse momentum. An additional loss of $25 \%$ is due to bremsstrahlung events that lead to a reduction of the mass by more than $100 \mathrm{MeV}$.

The $e^{+} e^{-}$invariant-mass spectrum corrected for efficiency and normalized to the number of charged particles in the acceptance [26] is shown in Fig. 2 together with the expectations from the hadron decay cocktail [28]. The $\phi$ meson yield in the $e^{+} e^{-}$channel is determined by integrating the invariant-mass spectra in the mass region between 0.9 and $1.1 \mathrm{GeV} / c^{2}$ in three transverse momentum bins. The $\rho$ meson could extend into this mass range if its spectral function is modified in the medium. Dileptons from the quark gluon plasma (QGP) phase also contribute to the physics background in this mass range. The sum of

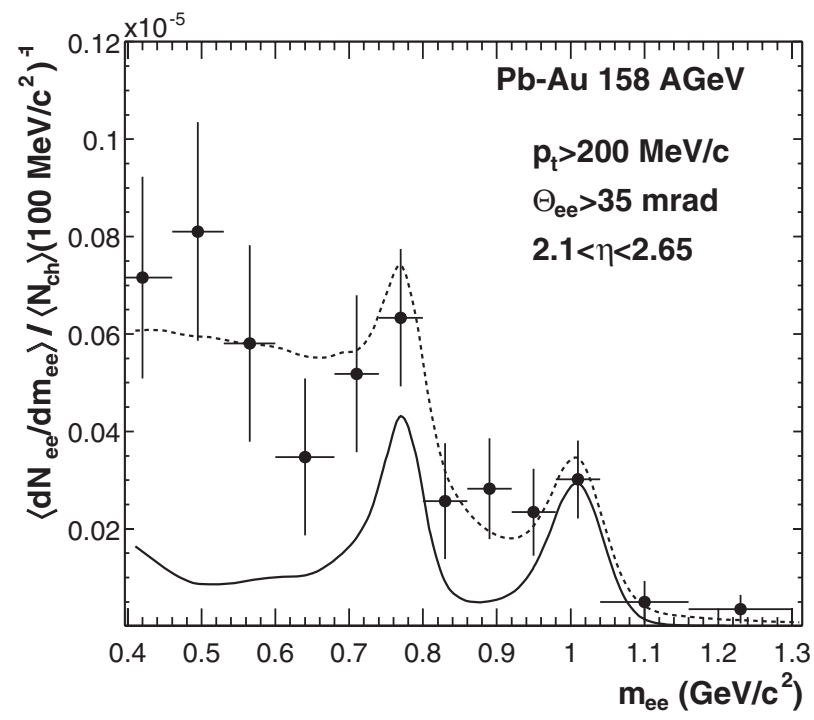

FIG. 2. Invariant-mass spectrum of $e^{+} e^{-}$pairs per charged particle compared to the hadron decay cocktail (thick solid line). Also plotted is a model calculation assuming in-medium spread $\rho$ width plus the dilepton yield from the QGP phase (dashed line) used to extract the physics background in the $\phi$ mass region. these two contributions is estimated to be $35 \%$ of the total yield in this mass region by inspecting theoretical models that include in-medium spreading of the $\rho$ width due to $2 \pi$ processes and the dilepton yield from the QGP phase [29]. If processes involving 4 or 6 pions would be included in the physics background could be somewhat larger [30]. Using another model [31] that also describes the CERES data gives a physics background contribution of $37 \%$. The measured $\phi$ yield has been corrected for the physics background assuming $35 \%$ for the three transverse momentum bins. We did not use a transverse momentum-dependent correction as there is no established prescription. We did, however, check various plausible scenarios for the transverse momentum dependence and find results well within the statistical errors quoted. A possible charm contribution that should be smaller than 3\% [32] has been neglected.

The efficiency- and acceptance-corrected $\phi$ meson yield is shown in Fig. 3 for both decay modes as a function of transverse momentum. The error bars shown are statistical. The systematic errors in the charged kaon analysis (plotted as boxes) contain contributions from the variation of the pair cuts, from the difference between the integral from the fit and from data points in the given interval, from the dependence of the yield on the fit window, and the function used to fit the residual background. The systematic errors in the dilepton analysis are $\pm 16 \%$. They contain the contribution from the analysis cuts, background substraction, and variation of mass range. They are not drawn because the errors in the dilepton channel are totally dominated by statistics.

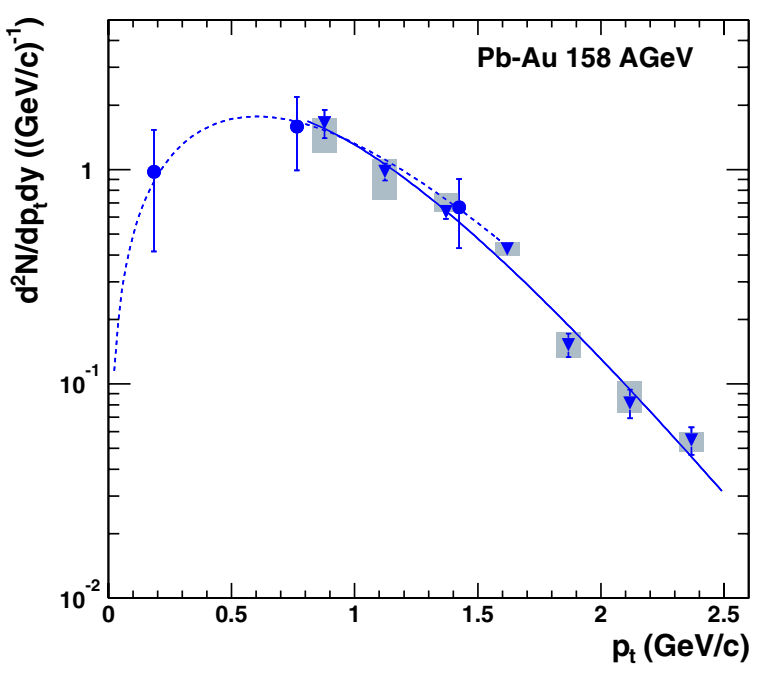

FIG. 3 (color online). Transverse momentum spectrum of $\phi$ mesons corrected for acceptance and efficiency reconstructed in the $e^{+} e^{-}$decay mode (circles) and in the $K^{+} K^{-}$decay channels (triangles). The spectrum in the $e^{+} e^{-}$decay mode has been scaled by 0.93 before comparing to the $K^{+} K^{-}$decay channels to account for the different rapidity intervals covered in the two analyses. Systematic errors in the kaon decay channel are shown as boxes. 


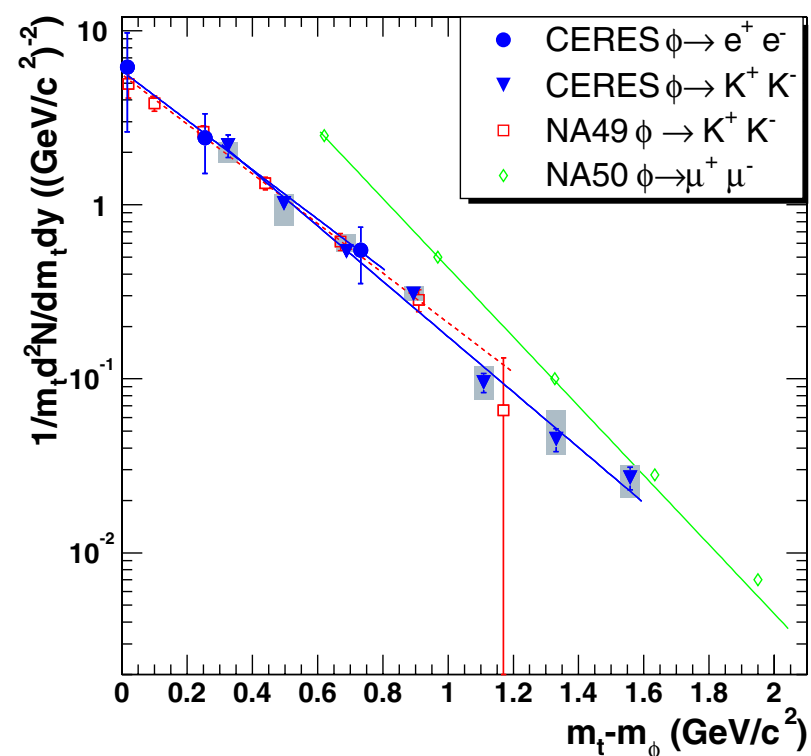

FIG. 4 (color online). Transverse mass distribution of $\phi$ mesons measured in the charged kaon (triangles) and in the dilepton (circles) decay mode after scaling (see text) compared to the results from NA49 (squares) and NA50 (diamonds). Error bars are statistical and systematic errors in the kaon channel are shown as boxes.

When the spectra are fitted with the function

$$
d^{2} N / d p_{t} d y=\frac{d N / d y}{T\left(T+m_{\phi}\right)} p_{t} \exp \left(-\frac{m_{t}-m_{\phi}}{T}\right)
$$

an inverse slope parameter of $T=273 \pm 9$ (stat) \pm 10 (syst) $\mathrm{MeV}$ and a rapidity density $d N / d y$ of $2.05 \pm$ 0.14 (stat) \pm 0.25 (syst) in the $K^{+} K^{-}$decay mode and $T=$ $306 \pm 82$ (stat) \pm 40 (syst) $\mathrm{MeV}$ and $d N / d y=2.19 \pm$ 0.52 (stat) \pm 0.34 (syst) in the dilepton decay mode are obtained averaging over their corresponding rapidity intervals. In order to compare the rapidity densities in the two decay channels the dilepton data are scaled by $\left(d N_{\phi} / d y\right)^{2-2.4}=0.93\left(d N_{\phi} / d y\right)^{2.1-2.65}$ [9]. The $\phi$ meson yields and inverse slope parameters obtained in both decay modes agree within the errors. A $\phi$ yield in the $e^{+} e^{-}$decay mode larger than 1.6 times the yield on the $K^{+} K^{-}$decay mode is excluded at $95 \%$ C.L. (statistical and systematic errors in both decay channels added in quadrature).

Moreover, the CERES results can be compared to the existing $\mathrm{Pb}-\mathrm{Pb}$ systematics [10] after accounting for the different measurement conditions. The NA49 measurement was done at $4 \%$ centrality and covered a rapidity range from 3 to 3.8 units [9]. We use the measured increase of charged hadron $\left(h^{-}\right)$multiplicity with centrality $\left(h_{4 \%}^{-} / h_{7 \%}^{-}=1.092 \pm 0.014\right)$ [21] to correct the $\phi$ meson yield for the different centralities between the two experiments. From the NA49 $\phi$ meson rapidity distribution a ratio of $(d N \phi / d y)^{3-3.8} /(d N \phi / d y)^{2-2.4}=1.07 \pm 0.11$ is obtained to account for the different rapidity coverage. Thus, a global scaling factor of $1.17 \pm 0.12$ is applied to the combined CERES data of Fig. 3 to make the comparison to the systematics of [10]. In Fig. 4 the $\phi$ transverse mass spectrum obtained by CERES after scaling is plotted together with the NA49 and NA50 data. The $\phi$ meson yields agree within the errors with the NA49 results, as does the yield in the $K^{+} K^{-}$channel when extrapolated down to $p_{t}=0$ using the measured inverse slope parameter. On the other hand, CERES data in the $K^{+} K^{-}$channel do not agree with NA50 results in the common $p_{t}$ region. This experiment, however, measures the leptonic channel. But, as stated above, in the CERES measurement the two decay modes agree. Possible differences of up to a maximum of $40 \%-50 \%$ as expected by models including only rescattering of the kaons [7], or of up to a maximum of $70 \%$ at the lowest $p_{t}\left(p_{t}<0.3 \mathrm{GeV} / c\right)$ expected by models including medium modifications of the $\phi$ mesons and kaons like the multiphase transport model (AMPT) [33], cannot be ruled out by the CERES results.

In conclusion, $\phi$ meson production has been measured in the dileptonic and hadronic decay channels in one experiment for the first time at the CERN SPS. The yields and the inverse slope parameters in both decay modes agree within the errors. The results reported here are in agreement with those from NA49 measured in the $\mathrm{K}^{+} \mathrm{K}^{-}$ channel. In the $e^{+} e^{-}$decay mode the yield agrees within errors and, as an upper limit, a value in excess of 1.6 times the yield in the $\mathrm{K}^{+} \mathrm{K}^{-}$is excluded at $95 \%$ C.L. Hence, the large discrepancy observed previously between the $\mu^{+} \mu^{-}$ and $K^{+} K^{-}$decay modes is not observed in the present CERES data. The theoretical predictions of small modifications as in $[7,33]$ are consistent with the present data; within the errors of the experiment we are not sensitive to the differences between these effects.

The CERES Collaboration acknowledges the good performance of the CERN PS and SPS accelerators as well as the support from the EST division. We would like to thank R. Campagnolo, L. Musa, A. Przybyla, W. Seipp, and B. Windelband for their contribution during construction and commissioning of the TPC and during data taking. We are grateful for excellent support by the CERN IT division for the central data recording and data processing. This work was supported by GSI, Darmstadt, the German BMBF, the Helmholtz Gemeinschaft Virtual Institue VH-VI 146, the US DOE, the Israeli Science Foundation, the MINERVA Foundation, and the Czech Science Foundation Contract No. 202/03/0879.

[1] See, e.g., Proceedings of the 17th International Conference on Ultra-Relativistic Nucleus-Nucleus Collisions, Oakland, California, USA, 11-17 January 2004, edited by H. G. Ritter and X.-N. Wang [J. Phys. G, 30 (2004)].

[2] F. Karsch, J. Phys. G 31, S633 (2005); Nucl. Phys. A698, 199c (2002).

[3] P. Braun-Munzinger, J. Stachel, and C. Wetterich, Phys. Lett. B 596, 61 (2004). 
[4] P. Koch, B. Müller, and J. Rafelski, Phys. Rep. 142, 167 (1986).

[5] D. Lissauer and E. V. Shuryak, Phys. Lett. B 253, 15 (1991).

[6] F. Klingl, T. Waas, and W. Weise, Phys. Lett. B 431, 254 (1998).

[7] S. C. Johnson, B. V. Jacak, and A. Drees, Eur. Phys. J. C 18, 645 (2001).

[8] N. Willis et al. (NA50 Collaboration), Nucl. Phys. A661, 534c (1999); B. Alessandro et al., Phys. Lett. B 555, 147 (2003).

[9] S. V. Afanasiev et al. (NA49 Collaboration), Phys. Lett. B 491, 59 (2000).

[10] D. Röhrich, J. Phys. G 27, 355 (2001).

[11] Addendum to proposal SPSLC/P280: CERN Report No. CERN/SPSLC 96-35/P280 Add.1, 1996 (unpublished).

[12] Technical Note on the NA45/CERES upgrade. CERN Report No. CERN/SPSLC 96-50, 1996 (unpublished).

[13] A. Marín for the (CERES Collaboration), Nucl. Phys. A661, 673c (1999).

[14] A. Marín et al. (CERES Collaboration), J. Phys. G 30, S709 (2004).

[15] G. Agakichiev et al. (CERES Collaboration), Eur. Phys. J. C 4, 231 (1998).

[16] G. Agakichiev et al. (CERES Collaboration), Phys. Rev. Lett. 75, 1272 (1995).

[17] G. Agakichiev et al. (CERES Collaboration), Phys. Lett. B 422, 405 (1998); B. Lenkeit for the CERES Collaboration, Nucl. Phys. A661, 23c (1999); J.P. Wessels for the CERES Collaboration, Nucl. Phys. A715, 262c (2003).
[18] G. Agakichiev et al. (CERES Collaboration), Eur. Phys. J. C 41, 475 (2005).

[19] D. Adamova et al. (CERES Collaboration), Phys. Rev. Lett. 91, 042301 (2003).

[20] J. Podolanski and R. Armenteros, Philos. Mag. 45, 13 (1954).

[21] CERES Collaboration, "Reconstruction of $\phi$ Mesons in the $K^{+} K^{-}$Channel" (to be published).

[22] S. Eidelmal et al., Phys. Lett. B 592, 1 (2004).

[23] O. Busch, Doctoral thesis, TU Darmstadt, 2006 (to be published).

[24] S. Yurevich, Doctoral thesis, University of Heidelberg, 2006.

[25] A. Cherlin, Doctoral thesis, Weizmann Institute of Science, 2005.

[26] D. Miśkowiec for the CERES Collaboration, nucl-ex/ 0511010 [Nucl. Phys. A (to be published)].

[27] GEANT, Detector Description and Simulation Tool, CERN Program Library Long Writeup W5013, 1995 (unpublished).

[28] H. Sako for the CERES Collaboration, GSI Technical Report No. 03-25, 2000 (unpublished).

[29] R. Rapp and J. Wambach, Adv. Nucl. Phys. 25, 1 (2000); E. Braaten, R. D. Pisarski, and T.-C. Yuan, Phys. Rev. Lett. 64, 2242 (1990).

[30] R. Rapp (private communication).

[31] K. Gallmeister et al., Nucl. Phys. A688, 939 (2001); B. Kämpfer (private communication).

[32] P. Braun-Munzinger, D. Miśkowiec, A. Drees, and C. Lourenço, Eur. Phys. J. C 1, 123 (1998).

[33] S. Pal, C. M. Ko, and Z. W. Lin, Nucl. Phys. A707, 525 (2002). 